\title{
Our expert highlights the most important research articles across the spectrum of topics relevant to the field of hepatic oncology
}

Expert: William Sanchez, Mayo Clinic College of Medicine, 200 First Street SW, Rochester, MN 55901, USA

Golfieri R, Bibao Jl, Carpanese L et al. Comparison of the survival and tolerability of radioembolization in elderly vs. younger patients with unresectable hepatocellular carcinoma. J. Hepatol. 59(4), 753-761 (2013).

The intersection of the increasing incidence of hepatocellular carcinoma (HCC) with an aging population leads to important questions regarding the best course of action for managing advanced-stage liver cancers in the geriatric population. This is particularly important when considering the significant expense and potential toxicities of therapies, such as yttrium-90 (Y90) radioembolization.

Golfieri and colleagues, on behalf of the European Network on Radioembolization with Yttrium-90 Resin Microspheres Study Group, performed a retrospective analysis of 325 consecutive patients at eight European centers treated for HCC with Y90 radioembolization over a 7-year period (2003-2009). The cohort was divided into two groups: those less than 70 years of age (younger, mean age: 58 years; $n=197$ ) and those greater than 70 years of age (older, mean age: 74 years; $n=128$ ). Both groups contained patients with advanced disease (the majority of patients with Barcelona Clinic Liver Cancer [BCLC] stage C HCC) who had progressed despite therapy or who were not candidates for other forms of locoregional therapy (LRT), such as transarterial chemoembolization. The groups were also similar in terms of gender (80-83\% male), etiology of liver disease (54-59\% viral cirrhosis) and severity of liver disease (81-85\% Child-Pugh class A).
Overall, Y90 radioembolization was equally well tolerated in both groups. Treatment-related mortality was low (three definitely related, 11 probably related), and common adverse events (e.g., fatigue, nausea, abdominal pain and fever) were similar in both groups. Survival analysis did not reveal any significant difference between the younger and older cohorts (median survival: 12.8 vs 14.5 months, respectively) and predominantly related to BCLC stage at the time of treatment.

The work of the authors reinforces that the major predictor of outcomes among patients with advanced-stage HCC is tumor stage and liver function. While this type of retrospective analysis is subject to bias (e.g., less robust elder patients may have been less likely to be referred for aggressive therapy), it does support the use of $\mathrm{Y} 90$ for the treatment of advanced-stage HCC among older patients.

Lai Q, Avolio AW, Graziadei I et al. Alpha-fetoprotein and modified response evaluation criteria in solid tumors progression after locoregional therapy as predictors of hepatocellular cancer recurrence and death after transplantation. Liver Transpl. 19(10), 1108-1118 (2013).

Selecting patients with a low risk of HCC recurrence after liver transplantation (LT) is vitally important in the setting of a critical shortage of suitable donor organs. While the Milan criteria identifies patients with a low risk of post-LT recurrence, it does not incorporate measures of pre-LT treatment response. In addition, there are patients with tumor burden beyond criteria

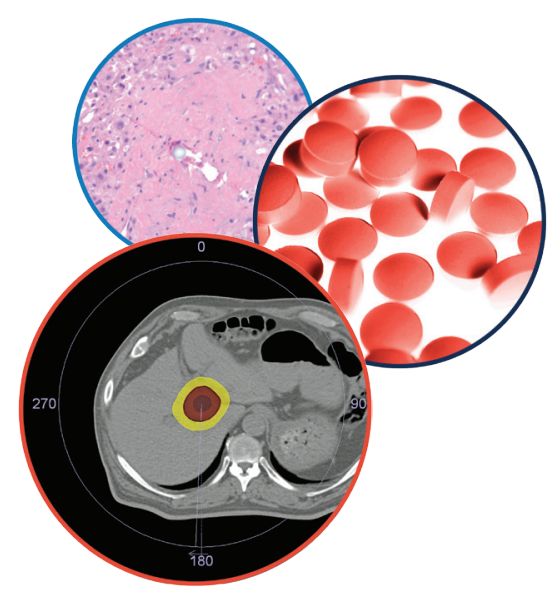

News \& Views

News

Journal Watch

Interview
Future 
who, after responding to LRT, may have satisfactory outcomes after transplantation.

Lai and colleagues, on behalf of the European Hepatocellular Cancer Liver Transplant Study Group, present an analysis of 422 patients with HCC who underwent LRT followed by LT at six European transplant centers over an 12-year period (19992010). The cohort contained 306 patients within Milan criteria and 116 beyond Milan criteria. The median age of LT recipients was 60 years and, not unexpectedly, the majority were male $(83 \%)$ and had viral-related liver disease (62\%). Transarterial chemoembolization was the most commonly performed form of LRT, having been used in $82 \%$ of patients either alone or in combination with other forms of LRT. The approach to LRT was different among patients within Milan criteria (where it was used as a bridge to transplantation) and those patients with tumor burden beyond Milan criteria (where LRT was typically used in a downstaging strategy). The patients underwent regular follow-up at their transplant center with imaging and $\alpha$-fetoprotein (AFP) measurements in the post-LT period.

The overall rates of HCC recurrence was low (14.5\%) and tumors typically recurred between 1 and 2 years post-LT (median time to recurrence: 16.6 months), in keeping with other published reports. Not unexpectedly, the recurrence rate was somewhat higher among patients transplanted for tumor burden beyond the Milan criteria (21.6\% recurrence vs $11.8 \%$ recurrence for patients within the Milan criteria).
Importantly, the authors identified that either biochemical (increasing AFP with a slope of greater than $15 \mathrm{ng} / \mathrm{ml} / \mathrm{month}$ ) or radiographical (progression per modified response evaluation criteria in solid tumors [mRECIST] criteria) following LRT were important and independent predictors of post-LT recurrence and death $(\mathrm{p}<0.001$ for both).

This study reinforces the importance of assessing HCC as a dynamic entity rather than a static one among patients awaiting LT. Clinically, many transplant physicians recognize that patients with aggressive tumor biology are at high risk for poor outcomes. With the increasing waitlist times for patients awaiting LT for HCC, identifying those patients who are unlikely to benefit from this form of aggressive surgery becomes increasingly important.

\section{Wong GLH, Chan HLY, Tse YK et al.}

On-treatment alpha-fetoprotein is a specific tumor marker for hepatocellular carcinoma in patients with chronic hepatitis $B$ receiving entecavir. Hepatology doi:10.1002/ hep.26739 (2013) (Epub ahead of print).

The use of AFP as a tumor marker is fraught with difficulties due to its lack of sensitivity and specificity. AFP levels are subject to false-positive elevations in the setting of active hepatitis. This is problematic given the dominant role of chronic hepatitis B virus in global HCC risk.
Wong and colleagues address this clinical challenge in part in their upcoming report in Hepatology (Epub ahead of print). In a retrospective analysis of 1531 hepatitis $B$ virus patients receiving antiviral therapy with entecavir at a single center in Hong Kong, the authors found that AFP levels started to increase 6 months before HCC was diagnosed and the area under the receiver operating curve for AFP was highest at the time of HCC diagnosis. Still, the sensitivity and specificity of AFP (using a low cutoff value of $6 \mathrm{~g} / \mathrm{ml}$ was only 80.7 and $80.4 \%$, respectively).

It is likely that the effect of antiviral therapy with the potent agent entecavir reduced hepatic necroinflammatory activity and accordingly reduced false-positive elevations in AFP (in addition to modulating the risk for HCC itself). While it is reassuring to know that AFP has reasonable performance characteristics in this setting, AFP by itself is still not sufficiently effective for HCC screening and would likely be substantially less reliable in patients with untreated viral hepatitis.

Financial \& competing interests disclosure The author has no relevant affiliations or financial involvement with any organization or entity with a financial interest in or financial conflict with the subject matter or materials discussed in the manuscript. This includes employment, consultancies, honoraria, stock ownership or options, expert testimony, grants or patents received or pending, or royalties.

No writing assistance was utilized in the production of this manuscript.

Journal Watch highlights some of the most important papers recently published in the field of hepatic oncology and research. The editorial team welcomes recommendations for relevant papers for inclusion in future issues.

Please direct your suggestions to:

Laura Dormer, Editor, Hepatic Oncology

I.dormer@futuremedicine.com 